\title{
Improved Newton Iteration for Integral Roots*
}

\author{
By Richard F. King**
}

\begin{abstract}
An improved Newton iteration procedure for computing $p$ th roots from best Chebyshev or Moursund initial approximations is developed. It differs from the usual Newton method by a multiplicative factor at each step. This multiplier halves the relative error by translating the usual one-sided error curve into a two-sided one, and then adjusting to make a Moursund-like fit. The generalized logarithmic error is used in determining this set of factors.
\end{abstract}

Introduction. The concept of generalized logarithmic error was introduced by Phillips [6] so that best Chebyshev fits to the $p$ th root could be readily transformed into best Moursund fits [1] for more accurate Newton iteration. And Taylor [8] independently showed the existence of these transformations for finding rational Moursund fits to $x^{\alpha}, 0<\alpha<1$. Such procedures correspond to exploiting the usual logarithmic error for the square root case [2], [3], [4]. In another paper [5], Ninomiya has shown how Newton iterates of Moursund fits for the square root can be improved from one-sided Chebyshev-like fits to Moursund two-sided fits for the next iteration; each improved fit has a maximum relative error that is only about half that of the one-sided fit. By using the generalized logarithmic error, we will derive similar improved two-sided fits for the $p$ th root, with $p$ being any positive integer; these fits in turn will form the basis for an improved Newton iteration method to compute integral roots.

Improvement of Iterated Approximations. Suppose that after $n$ steps $(n=$ $0,1,2, \cdots)$ of some iteration procedure, we have arrived at an approximation $y_{n}(x)$ to the $p$ th root of $x$. The initial approximation $y_{0}(x)$ is for $x$ given on a positive interval $[a, b]$. For example, $y_{n}$ could be the result of the successive application of the usual Newton iteration formula

$$
y_{n}=\frac{1}{p}\left([p-1] y_{n-1}+\frac{x}{y_{n-1}^{p-1}}\right), \quad(p=2,3,4, \cdots) .
$$

Further, presume that the relative error,

$$
\delta_{n}(x)=\frac{y_{n}(x)-x^{1 / p}}{x^{1 / p}},
$$

Received January 8, 1970.

AMS 1969 subject classifications. Primary 6510, 6520, 6550.

Key words and phrases. Newton's method, integral root, generalized logarithmic error, one-sided error, Moursund approximation, best rational fit, Chebyshev-like error, improvement factors, convergence rate.

* Work supported by the British Science Research Council at the University of Dundee, and by the U. S. Atomic Energy Commission.

** Present address: Argonne National Laboratory, Argonne, Illinois 60439.

Copyright (c) 1971, American Mathematical Society 
is a one-sided Chebyshev-like error. By this we mean that $\delta_{n}(x)$ is continuous and assumes alternate maximum values $\sigma_{n}$ and minimum values 0 . Similarly, we say a two-sided Chebyshev-like error $\bar{\delta}_{n}(x)$ is one that assumes alternate maximum values $\bar{\sigma}_{n}$ and minimum values $\left(-\bar{\sigma}_{n}\right)$.

The prescription in the following theorem enables us to improve on the fit $y_{n}$.

THEOREM. Let $y_{n}$ be an approximation on $[a, b]$ to the pth root of $x$, and let its relative error curve be one-sided and Chebyshev-like, with maximum value $\sigma_{n}$. Then the approximation $\bar{y}_{n}(x)$ defined by

$$
\bar{y}_{n}=\left(\frac{2}{2+\sigma_{n}}\right) y_{n}
$$

has a relative error that is two-sided and Chebyshev-like, and the maximum relative error is

$$
\bar{\sigma}_{n}=\frac{\sigma_{n}}{2+\sigma_{n}}
$$

Proof. First, translate the relative error curve $\delta_{n t}$ for $y_{n}$ by $\left(-\sigma_{n} / 2\right)$ :

$$
\delta_{n}-\frac{\sigma_{n}}{2}=\frac{y_{n}-x^{1 / p}}{x^{1 / p}}-\frac{\sigma_{n}}{2}=\frac{y_{n}-\left(1+\sigma_{n} / 2\right) x^{1 / p}}{x^{1 / p}} .
$$

Hence,

$$
\left\{\frac{\delta_{n}-\sigma_{n} / 2}{1+\sigma_{n} / 2}\right\}=\left\{\frac{y_{n} /\left(1+\sigma_{n} / 2\right)-x^{1 / p}}{x^{1 / p}}\right\},
$$

so that $\bar{y}_{n}=y_{n} /\left(1+\sigma_{n} / 2\right)$ is an approximation to $x^{1 / p}$, and furthermore, it has a two-sided Chebyshev-like relative error $\bar{\delta}_{n}=\left(\delta_{n}-\sigma_{n} / 2\right) /\left(1+\sigma_{n} / 2\right)$. To see this, note that $\bar{\delta}_{n}$ is merely a translation of $\delta_{n}$ followed by a contraction. Thus, maxima and minima remain respectively maxima and minima. Finally, a maximum $x_{1}$ of $\delta_{n}$ becomes a maximum of $\bar{\delta}_{n}$ with value

$$
\bar{\sigma}_{n}=\bar{\delta}_{n}\left(x_{1}\right)=\frac{\sigma_{n}-\sigma_{n} / 2}{1+\sigma_{n} / 2}=\frac{\sigma_{n}}{2+\sigma_{n}} ;
$$

also, a zero (minimum) $x_{0}$ of $\delta_{n}$ becomes a minimum of $\bar{\delta}_{n}$ with value

$$
\bar{\delta}_{n}\left(x_{0}\right)=\frac{0-\sigma_{n} / 2}{1+\sigma_{n} / 2}=-\frac{\sigma_{n}}{2+\sigma_{n}}=-\bar{\sigma}_{n} .
$$

This completes the proof.

But both Phillips and Taylor have shown that such a two-sided Chebyshev-like approximation $\bar{y}_{n}$ becomes a Moursund-like approximation $\hat{y}_{n}$ by the transformation

$$
\hat{y}_{n}=k_{n} \bar{y}_{n}
$$

for

(5) $\quad k_{n}=\frac{1}{1+\bar{\sigma}_{n}}\left\{\frac{1}{p-1}\left[\left(\frac{1+\bar{\sigma}_{n}}{1-\bar{\sigma}_{n}}\right)^{p-1}+\left(\frac{1+\bar{\sigma}_{n}}{1-\bar{\sigma}_{n}}\right)^{p-2}+\cdots+\left(\frac{1+\bar{\sigma}_{n}}{1-\bar{\sigma}_{n}}\right)\right]\right\}^{1 / p}$.

That is, the Newton iterate of $\hat{y}_{n}$ has a one-sided Chebyshev-like relative error curve.

Combining (2) and (4) and using (3), we get a Moursund-like approximation $\hat{y}_{n}$ from a one-sided Chebyshev-like fit $y_{n}$ by the transformation 
(6)

$$
\begin{aligned}
\hat{y}_{n} & =\frac{2 k_{n} y_{n}}{2+\sigma_{n}} \\
& =\frac{y_{n}}{1+\sigma_{n}}\left\{\frac{1}{p-1}\left[\left(1+\sigma_{n}\right)^{p-1}+\left(1+\sigma_{n}\right)^{p-2}+\cdots+\left(1+\sigma_{n}\right)\right]\right\}^{1 / \nu} .
\end{aligned}
$$

The improved maximum relative error $\hat{\sigma}_{n}$ is given by

$$
\hat{\sigma}_{n}=k_{n}\left(1+\bar{\sigma}_{n}\right)-1=k_{n}\left(\frac{2+2 \sigma_{n}}{2+\sigma_{n}}\right)-1 \text {. }
$$

With the idea of improving the Newton iterate at every step by the transformation (6), we define the improvement factors $C_{n}$ by

$$
C_{n}=2 k_{n} /\left(2+\sigma_{n}\right) \text {. }
$$

Note that, for $p=2, C_{n}=1 /\left(1+\sigma_{n}\right)^{1 / 2}$ is the correcting factor used by Ninomiya in his improved Newton iteration scheme.

Recurrence Formulas for Improvement Factors. Let us try to find a recursion relation for the multipliers

$$
C_{n}=\frac{1}{1+\sigma_{n}}\left\{\frac{1}{p-1}\left[\left(1+\sigma_{n}\right)^{p-1}+\left(1+\sigma_{n}\right)^{p-2}+\cdots+\left(1+\sigma_{n}\right)\right]\right\}^{1 / p} .
$$

Newton iteration from $\hat{y}_{n}$ to $y_{n+1}$ yields, by (1),

$$
\begin{aligned}
1+\delta_{n+1}=\frac{y_{n+1}}{x^{1 / p}} & =\frac{1}{p}\left([p-1] \frac{\hat{y}_{n}}{x^{1 / p}}+\frac{\left(x^{1 / p}\right)^{p-1}}{\hat{y}_{n}^{p-1}}\right) \\
& =\frac{1}{p}\left([p-1]\left[1+\hat{\delta}_{n}\right]+\frac{1}{\left[1+\hat{\delta}_{n}\right]^{p-1}}\right),
\end{aligned}
$$

where $\hat{\delta}_{n}$ is the relative error for approximation $\hat{y}_{n}$ to $x^{1 / \nu}$. Consequently,

$$
1+\sigma_{n+1}=\frac{1}{p}\left([p-1]\left[1+\hat{\sigma}_{n}\right]+\frac{1}{\left[1+\hat{\sigma}_{n}\right]^{p-1}}\right),
$$

where

$$
1+\hat{\sigma}_{n}=1+\max _{x \in[a, b]}\left|\frac{\hat{y}_{n}}{x^{1 / p}}-1\right|=\max _{x \in[a, b]}\left(\frac{C_{n} y_{n}}{x^{1 / p}}\right)=C_{n}\left(1+\sigma_{n}\right) .
$$

From (8), we have that

$$
\left(1+\sigma_{n}\right)^{p-1}=\frac{1}{(p-1) C_{n}^{p}}\left\{\left[1+\sigma_{n}\right]^{p-2}+\left[1+\sigma_{n}\right]^{p-3}+\cdots+1\right\} .
$$

Therefore,

$$
\begin{aligned}
1+\sigma_{n+1} & =\frac{1}{p}\left([p-1] C_{n}\left[1+\sigma_{n}\right]+\frac{1}{C_{n}^{p-1}\left[1+\sigma_{n}\right]^{p-1}}\right) \\
& =\frac{1}{p}\left([p-1] C_{n}\left[1+\sigma_{n}\right]+\frac{[p-1] C_{n}^{p}}{C_{n}^{p-1}\left\{\left[1+\sigma_{n}\right]^{p-2}+\left[1+\sigma_{n}\right]^{p-3}+\cdots+1\right\}}\right) \\
& =\left(\frac{p-1}{p}\right) C_{n}\left(\frac{\left[1+\sigma_{n}\right]^{p-1}+\left[1+\sigma_{n}\right]^{p-2}+\cdots+1}{\left[1+\sigma_{n}\right]^{p-2}+\left[1+\sigma_{n}\right]^{p-3}+\cdots+1}\right) \\
& =\left(\frac{p-1}{p}\right) C_{n}\left(\frac{\left[1+\sigma_{n}\right]^{p-1}}{\left[1+\sigma_{n}\right]^{p-2}+\left[1+\sigma_{n}\right]^{p-3}+\cdots+1}+1\right) .
\end{aligned}
$$


Applying (9) again,

$$
1+\sigma_{n+1}=\left(\frac{p-1}{p}\right) C_{n}\left(\frac{1}{[p-1] C_{n}^{p}}+1\right)=\frac{1+(p-1) C_{n}^{p}}{p C_{n}^{p-1}}
$$

Substituting (10) into (8) results in

$$
C_{n+1}=\left(\frac{1}{p-1}\left[D_{n}^{p-1}+D_{n}^{p-2}+\cdots+D_{n}\right]\right)^{1 / p},
$$

where

$$
D_{n}=\frac{p C_{n}^{p-1}}{1+(p-1) C_{n}^{p}} .
$$

This is the desired recurrence formula for the improvement factors $C_{n}$. The value of $C_{0}$, from (7) and (3), is

$$
C_{0}=\frac{2 k_{0}}{2+\sigma_{0}}=k_{0}\left(1-\bar{\sigma}_{0}\right),
$$

for an initial two-sided Chebyshev approximation $\bar{y}_{0}$ to $x^{1 / p}$.

Similarly, the factor $C_{n}^{*}=2 /\left(2+\sigma_{n}\right)$ in (2) that carries a one-sided Chebyshevlike fit into a two-sided one has the recurrence formula

$$
C_{n+1}^{*}=\frac{2}{1+\frac{1}{p}\left([p-1]\left[\frac{1}{p-1}\left(E_{n}^{p-1}+\cdots+E_{n}\right)\right]^{1 / p}+\left[\frac{1}{p-1}\left(E_{n}^{p-1}+\cdots+E_{n}\right)\right]^{-((p-1) / p]}\right)},
$$

where

$$
E_{n}=\frac{2-C_{n}^{*}}{C_{n}^{*}}, \quad C_{0}^{*}=\frac{2}{2+\sigma_{0}}=1-\bar{\sigma}_{0} .
$$

More conveniently, perhaps, $C_{n}^{*}$ can be generated from $C_{n-1}$ by

$$
C_{n}^{*}=\frac{2}{1+\left(1+[p-1] C_{n-1}^{p}\right) /\left(p C_{n-1}^{p-1}\right)} .
$$

Improved Newton Procedure. We thus have the basis for an improved Newton algorithm to obtain $x^{1 / p}$ on an interval $[a, b]$ from an initial best rational Chebyshev approximation $\bar{y}_{0}$, in the sense of relative error. The iteration formula is

$$
\hat{y}_{n}=\frac{C_{n}}{p}\left([p-1] \hat{y}_{n-1}+\frac{x}{\hat{y}_{n-1}^{p-1}}\right)
$$

for $n=1,2, \cdots, N-1$, with $C_{n}$ given by (11), (12), and (13). The Moursund approximation $\hat{y}_{0}(x)$ is derived from the Chebyshev fit $\bar{y}_{0}(x)$ by applying (from (5)) the multiplier

$$
k_{0}=\frac{1}{1+\bar{\sigma}_{0}}\left\{\frac{1}{p-1}\left[\left(\frac{1+\bar{\sigma}_{0}}{1-\bar{\sigma}_{0}}\right)^{p-1}+\cdots+\left(\frac{1+\bar{\sigma}_{0}}{1-\bar{\sigma}_{0}}\right)\right]\right\}^{1 / p} .
$$

That is to say, $\hat{y}_{0}(x)$ is a best generalized logarithmic error fit to $x^{1 / p}$. 
For the last step, as Ninomiya has pointed out, a two-sided Chebyshev-like fit should be obtained. Thus the final approximation is

$$
\bar{y}_{N}=\frac{C_{N}^{*}}{p}\left([p-1] \hat{y}_{N-1}+\frac{x}{\hat{y}_{N-1}^{p-1}}\right),
$$

for $C_{N}^{*}$ computed from (14) and (15), or (16).

In the square root case, best rational Moursund fits of the form $\left(P_{r} / Q_{r}\right)$ or $\left(P_{r+1} / Q_{r}\right)$ remain best Moursund fits with successive improved Newton iteration steps. (A best Moursund fit is one whose Newton iterate has a relative error with least maximum magnitude.) This nice property (see [5]) does not appear to hold for $p \geqq 3$, simply because $p$ th-root Newton iterates-even of the improved variety-do not have enough oscillations in their relative error curves. That is, it does not follow that an improved Newton approximant at a given stage becomes a best one-sided Chebyshev fit upon iteration.

Convergence Rate and Conclusion. As with the square root, this improved Newton procedure for $N$ steps converges by a factor of approximately $2^{2^{N}-1}$ faster than the usual Newton method. This improvement is at the negligible cost of storing and providing for the indexing of a small table of constants $\left(C_{n} / p\right)$. The only situation where this would not be attractive is that of the root $p$ also being the floating point base, as with $p=2$ on a binary machine. In this case, an exponential add order is much faster than a multiplication, and it is usually better just to use Newton's method, taking (if necessary) an extra step.

In the square root case, the question raised by Ninomiya as to whether the inequality

$$
\hat{\sigma}_{n}<\sigma_{n} / 2^{2^{n}-1}
$$

generally holds can be answered in the negative. For, consider the initial approximation $y_{0}=1$ to $\sqrt{ } x$ on $\left[\frac{1}{2}, 2\right]$. This is a best Moursund fit, as are all of its improved Newton iterates. The table below, based on the error recurrence relations

$$
\sigma_{n+1}=\frac{\sigma_{n}^{2}}{2\left(1+\sigma_{n}\right)}, \quad \hat{\sigma}_{n+1}=\left(1+\frac{\hat{\sigma}_{n}^{2}}{2\left(1+\hat{\sigma}_{n}\right)}\right)^{1 / 2}-1,
$$

shows that the inequality fails to hold for $n=3$.

\begin{tabular}{cccrc}
\hline$n$ & $\sigma_{n}$ & $\hat{\sigma}_{n}$ & $2^{2^{n-1}}$ & $\sigma_{n} \div \hat{\sigma}_{n}$ \\
\hline 0 & .414213562373 & .414213562373 & 1 & 1.0000 \\
1 & .060660171780 & .029883571953 & 2 & 2.0299 \\
2 & .001734606681 & .000216755350 & 8 & 8.0026 \\
3 & .000001501825 & .000000011743 & 128 & 127.89 \\
\hline
\end{tabular}

Department of Mathematics

University of Dundee

Dundee, Scotland 
1. DAvid G. Moursund, "Optimal starting values for Newton-Raphson calculation of $\sqrt{ } x$," Comm. $A C M$, v. 10, 1967, pp. 430-432. MR 39 \#2297.

2. Richard F. King \& David L. Phillips, "The logarithmic error and Newton's method for the square root," Comm. $A C M$, v. 12, 1969, pp. 87-88.

3. P. H. Sterbenz \& C. T. Fike, "Optimal starting approximations for Newton's method," Math. Comp., v. 23, 1969, pp. 313-318. MR 39 \#6511.

4. Ichizo NinomiYA, "Generalized rational Chebyshev approximation," Math. Comp., v. 24,1970 , pp. $159-169$.

5. ICHIzo NinOMIYA, "Best rational starting approximations and improved Newton iteration for the square root," Math. Comp., v. 24, 1970, pp. 391-404.

6. David L. Phillips, "Generalized logarithmic error and Newton's method for the $m$ th root," Math. Comp., v. 24, 1970, pp. 383-389.

7. D. G. MOURSUND \& G. D. TAYLOR, "Optimal starting values for the Newton-Raphson calculation of inverses of certain functions," SIAM J. Numer. Anal., v. 5, 1968, pp. 138-150. MR 37 \# 1074.

8. G, D. TAYlor, “Optimal starting approximations for Newton's method," J. Approximation Theory (To appear.) 